\title{
Article
}

\section{Novel critical point drying (CPD) based preparation and transmission electron microscopy (TEM) imaging of protein specific molecularly imprinted polymers (HydroMIPs)}

Hawkins, DM, Ellis, EA, Stevenson, D, Holzenburg, A and Reddy, Subrayal M

Available at http://clok.uclan.ac.uk/13679/

Hawkins, DM, Ellis, EA, Stevenson, D, Holzenburg, A and Reddy, Subrayal M ORCID: 0000-0002-7362-184X (2007) Novel critical point drying (CPD) based preparation and transmission electron microscopy (TEM) imaging of protein specific molecularly imprinted polymers (HydroMIPs). Journal of Materials Science, 42 (22). 9465 - 9468. ISSN 0022-2461

It is advisable to refer to the publisher's version if you intend to cite from the work. http://dx.doi.org/10.1007/s10853-007-1806-4

For more information about UCLan's research in this area go to http://www.uclan.ac.uk/researchgroups/ and search for <name of research Group>.

For information about Research generally at UCLan please go to http://www.uclan.ac.uk/research/

All outputs in CLoK are protected by Intellectual Property Rights law, including Copyright law. Copyright, IPR and Moral Rights for the works on this site are retained by the individual authors and/or other copyright owners. Terms and conditions for use of this material are defined in the policies page. 


\title{
The Novel Critical Point Drying (CPD) Based Preparation and Transmission Electron Microscopy (TEM) Imaging of Protein Specific Molecularly Imprinted Polymers (HydroMIPs)
}

\author{
Daniel M. Hawkins*, E. Ann Ellis ${ }^{\downarrow}$, Derek Stevenson*, \\ Andreas Holzenburg $^{\uparrow}$ \& Subrayal M. Reddy*
}

*School of Biomedical and Molecular Sciences, University of Surrey, Guildford, Surrey, GU2 7XH, UK;

${ }^{\uparrow}$ Microscopy \& Imaging Centre, Texas A\&M University, College Station, TX 77843, USA.

Corresponding Author: Tel: +44 (0)1483 68 6396, Fax: +44 (0)1483 686401 e-mail: s.reddy@surrey.ac.uk (S.M.Reddy) 


\begin{abstract}
We report the transmission electron microscopy (TEM) imaging of a hydrogel-based molecularly imprinted polymer (HydroMIP) specific to the template molecule bovine haemoglobin $(\mathrm{BHb})$. A novel critical point drying based sample preparation technique was employed to prepare the molecularly imprinted polymer (MIP) samples in a manner that would facilitate the use of TEM to image the imprinted cavities, and provide an appropriate degree of both magnification and resolution to image polymer architecture in the $<10 \mathrm{~nm}$ range. For the first time, polymer structure has been detailed that clearly displays molecularly imprinted cavities, ranging from 5-50 $\mathrm{nm}$ in size, that correlate (in terms of size) with the protein molecule employed as the imprinting template. The modified critical point drying sample preparation technique used may potentially play a key role in the imaging of all molecularly imprinted polymers, particularly those prepared in the aqueous phase.
\end{abstract}

\title{
Keywords
}

Molecular Imprinting, HydroMIPs, Hydrogels, Critical Point Drying, Transmission Electron Microscopy. 


\section{Introduction}

Molecular Imprinting (MI) is an analytical technique that holds much promise for the development of a variety of far reaching interdisciplinary fields. The principle of MI involves the formation of highly specific polymer cavities that can be engineered to display both specificity and selectivity for a vast range of differing molecules. The resulting polymer can be employed in many differing manners and applications, but it is its use as a selective, molecular recognition material in biosensor strategies that holds much promise for the development and progression of the technique into the clinical and commercial setting.

Molecularly imprinted polymers (MIPs) are formed following the copolymerisation of one or more functional monomers and a cross-linking agent, in the presence of a template molecule of interest. The template molecule is first allowed to associate with the functional monomer in an appropriate porogen (solvent), where an abundance of non-covalent interactions (including hydrogen-bonding and electrostatic interactions) occur to loosely form a hostguest complex. Upon polymerisation of the solution in the presence of a cross-linking agent, a rigid polymer matrix is formed within which the template molecule is entrapped. By removing the template molecule, typically by washing with a series of optimised elution solvents, a cavity is left that is complimentary in terms of shape, size and charge to that of the original template molecule.

To date, MI has failed to fully recognise its potential due to the reliance upon organic solvents to promote the host-guest interactions that govern the technique. This has resulted in the inherent inability to devise imprinting protocols for biomolecules of clinical significance. Synthesis in aqueous media of chemically and mechanically stable MIPs, that demonstrate specific recognition of biomolecules continues to be a significant challenge, as aqueous 
solutions significantly reduce the binding strength of the non-covalent template-monomer interactions that are integral to the production of an imprinting effect. However, hydrogels are beginning to gain popularity as suitable aqueous based matrices for imprinting protocols, particularly following the reported use of polyacrylamide as a successful imprinting matrix [1-3]. Subsequently, we have extensively optimised and investigated the development of polyacrylamide hydrogels, or HydroMIPs for MI [4].

Considering both the rapidly progressing nature and the depth of knowledge surrounding MI, it is surprising that little work has been carried out to actually image the molecular cavities that form when making a MIP. Characterisation of MIPs is often performed by techniques such as HPLC and spectrophotometry, but as these techniques do not allow us to physically visualise the cavities that are being formed, many assumptions still underpin our current knowledge of MI. To our knowledge, direct visualisation of molecularly imprinted cavities has never been reported.

Transmission electron microscopy (TEM) is by far the most preferred technique for imaging HydroMIPs, as it offers both the resolution and magnification necessary to accurately image the imprinted cavities. However, due to the aqueous nature of the hydrogels, it is vital that they are imaged in a fully dehydrated form, therefore facilitating the use of TEM. To date, MIPs have been characterised using a variety of imaging techniques, including various differing forms of electron microscopy (EM) [5-8]. EM has also been succesfully employed to image polyacrylamide hydrogels following extensive and often time consuming sample preparation techniques such as vacuum drying [9-11], freeze drying [12], critical point drying [13], and sequential solvent dehydration and epoxy embedding [14]. Many of the previous attempts to image hydrogels using EM have produced poor images that lack structural detail. 
This has occurred invariably as a direct result of inappropriate sample preparation techniques and an alternative approach to the preparation of hydrogel-based samples is urgently required to allow visual characterisation of the polymer materials in their natural form.

Critical point drying (CPD) is a technique used to dry samples that are typically very fragile or wet, without deforming or collapsing the structure, and commonly finds applications in scanning electron microscopy. CPD avoids the sample degradation that occurs in air or vacuum drying processes, and eliminates the drying artefacts that are now, unacceptable when considering the multitude of sample preparation techniques available. CPD methods avoid sample damage by never allowing a liquid/gas interface to develop, which in turn prevents sample exposure to the large surface tension forces that may cause the structure within a sample material to collapse. CPD is often performed with a specific CPD apparatus and involves a considerable investment of time. The method employed here is a modification of a rapid, simplified chemical CPD procedure [15].

In this paper, we describe a highly novel cryo sample preparation method that allows the visualisation of protein specific cavities for a polyacrylamide HydroMIP that has been engineered for the template molecule bovine haemoglobin $(\mathrm{BHb})$. We detail the HydroMIP in its native form with protein entrapped within the polymer matrix, and also following template elution. The findings further contribute to the understanding of aqueous phase molecular imprinting protocols. 


\section{Materials and Methods}

\section{HydroMIP and HydroNIP Production}

Polyacrylamide HydroMIPs and HydroNIP controls were made and granulated as previously described by the authors [4]. Following granulation, HydroMIP samples were treated in three differing manners. MIP 1 gels refer to MIPs produced with protein still entrapped. The latter were washed with five $2 \mathrm{ml}$ volumes of deionised (DI) water. MIP 2 gels refer to MIPs have protein removal, leaving cavities. MIP 2 gels were washed with five $2 \mathrm{ml}$ volumes of DI water followed by five $2 \mathrm{ml}$ volumes of SDS:AcOH eluant. Each wash/elution step was performed by centrifugation at $3000 \mathrm{rpm}$ for 5 mins using a Centaur II centrifuge (Fisher Scientific, Loughborough, UK).

\section{Sample Prep for TEM}

A 1:1 dilution in DI water of all HydroMIPs and HydroNIPs was made. $1 \mu 1$ aliquots of gel suspensions were pipetted onto 400 mesh, carbon stabilised, Formvar coated glow discharged copper TEM grids. The grids were firmly grasped with forceps, and plunged into liquid nitrogen. Following constant agitation of the sample in the liquid nitrogen for approximately 30 secs, the grid was then transferred to $100 \%$ methanol and agitated for approximately 20 secs. The grid was then transferred to hexamethyldisilizane (HMDS) (Electron Microscopy Sciences, Hatfield, PA) and again agitated for approximately 20 secs. The samples were then left in the fume hood for 30 mins to allow the HMDS to volatize off. Grids were examined in a JEOL 1200EX TEM at an accelerating voltage of $100 \mathrm{kV}$. Magnification was calibrated with a 2,160 ln/inch diffraction grating replica (Electron Microscopy Sciences, Hatfield, PA).

\section{Results and Discussion}

The studies conducted detail the imaging of molecularly imprinted polymer gels in three differing physical forms. "NIP" refers to the non-imprinted polymer. MIP 1 refers to the 
BHb-specific HydroMIP in its natural form - the gel has been cast in the presence of the template protein, which has not been eluted in any manner and theoretically remains entrapped within the cavity/cavities of the polymer matrix. The physical form of MIP 2 potentially offers the most indicative information regarding the presence of protein specific cavities. The template molecule has been removed from the gel, and the differences in form should be clearly notable in comparison to MIP 1

The size of a BHb molecule is approximately $55 \AA$ in diameter. It is therefore reasonable to assume that the minimum cavity diameter that we could expect to find will be in the region of $5.5 \mathrm{~nm}$, which equates to one protein molecule to one cavity. However, protein-protein interactions occur widely and are generally favoured under conditions that reduce the net charge on the molecules. As the imprinted polymer is aqueous based with water being the main constituent of the material, it is also reasonable to assume that larger cavities will also be evident that will be specific to larger groups of $\mathrm{BHb}$ molecules, and will have formed as a result of protein-protein interactions occurring in the pre-polymeric solution.

Initial attempts to prepare the sample in an appropriate manner for TEM analysis involved the sequential dehydration of the polyacrylamide hydrogels, followed by embedding in epoxy resin and the post-staining of ultra thin sections with either uranyl acetate, ruthenium tetroxide, osmium tetroxide or phosphotungstic acid. The sample preparation was particularly laborious, and sectioning of the material proved particularly problematic if the dehydration had not been $100 \%$ successful. It was also evident that all post staining methodologies produced excessively stained samples with a great deal of staining artefacts, even with minimal exposure to the stain. Although hydrogel structure was evident in all cases, interpretation of these structures was very difficult due to the visual occlusion caused 
by the heavy metal stains. It was noted however, that when no post stain was utilised, the hydrogels exhibited excellent contrast and natural electron density. This suggested that if an alternative preparation methodology could be employed to preserve the structure of the samples, a post staining stage would not be necessary. In addition, samples can shrink during dehydration and resin polymerisation and ultramicrotomy can possibly disrupt the ultrafine structure network of hydrogels. Cryo-transmission electron microscopy has been proposed as the optimal sample preparation method for hydrogels [17]; however, only a few electron microscopy facilities are equipped with cryo-TEMs. The modified cryogenic CPD methodology that was employed, although simple in its approach, allowed the quick and reproducible preparation of samples in a manner that is highly applicable and affordable in any microscopy facility.

Figure 1 shows an image of the non-imprinted NIP and there is no significant ultrastructural detail. Figure 2 shows an image of the molecularly imprinted polymer, with protein still in place (MIP 1). The polymer sample clearly exhibits a great deal of natural electron density, as no post stain has been applied to enhance the image. The form of the structure correlates closely with images of highly crosslinked polymer hydrogels that have been reported before $[12,13,16]$. At various points across the gel structure, lighter areas of lesser electron density are reproducibly apparent that are 5-10 $\mathrm{nm}$ in diameter. The HydroMIP in this form still has the template protein embedded within its polymer matrix. However, the degree to which the template is bound within the HydroMIP matrix will differ depending upon the strength and number of non-covalent interactions that formed during both the template-monomer association stage and free radical polymerisation. It is therefore plausible that these areas are in fact free cavities that have been exposed as a result of the multiple DI washes that were employed to remove any unreacted or unbound template from the material. 
Figure 3. shows an image of MIP 2 Hydrogel. The structure again demonstrates a great deal of natural electron density and contrast, and the crosslinked nature of the polymer matrix is clearly defined. The large spherical lobe-like structures represent the voids that were occupied by the aqueous content of the gel, which have been successfully preserved by the cryogenic preparation process. This image clearly differs from that of MIP 1 (Fig 2). Throughout the body of the HydroMIP matrix, small spherical areas of lesser electron density, identical to those observed and detailed in MIP 1 are apparent. However, they are in abundance throughout the body of the gel matrix, and are reproducibly in the region of 5-10 $\mathrm{nm}$ in diameter. We believe that this structural detail is clearly indicative of imprinted cavities that have been exposed as a direct result of denaturing and removing the template molecule with the elution solvent.

\section{Conclusions}

We have described a simple, novel and effective critical point drying based sample preparation methodology for the TEM imaging of protein specific molecularly imprinted polymer hydrogels. The images obtained clearly show that when the hydrogels are prepared by this technique, the structure of the sample is preserved, and a natural electron density is exhibited. Clear distinctions in polymer structure can be made between all MIP forms, which in turn is indicative of molecularly imprinted cavities in the MIP 2 sample, and not in MIP's 1 and 3. For the first time, data is presented to physically show the evidence of imprinted cavities within a polymer matrix, and this sample preparation methodology holds much promise for future high- resolution imaging of molecular imprinted polymers, which in turn will further elucidate substantial and much needed information detailing polymer structure. 


\section{Acknowledgements}

The authors would like to gratefully acknowledge the financial support provided by the

Department of Trade and Industry and the Engineering and Physical Sciences Research Council.

\section{References}

1. J.L. LIAO, Y. WANG and S. HJERTEN, Chromatographia 42 (1996) 359.

2. S. HJERTEN, J.L. LIAO, K. NAKAZATO, Y. WANG, G. ZAMARATSKAIA and H. X. ZHANG (1997) Chromatographia 44 (1997) 227.

3. D. TONG, C. HETENYI, Z. BIKADI, J. P. GAO, and S. HJERTEN (2001) Chromatographia 54 (2001) 7.

4. D. M. HAWKINS, D. STEVENSON and S. M. REDDY (2005) Anal. Chim. Acta 542 (2005) 61.

5. B. RUCKER and U. KOLB, Micron 36 (2005) 247.

6. Z. H. ZHANG, Y. M. LONG, Y. J. LIU, and S. Z. YAO, Instrumen. Sci. Tech. 32 (2004) 507.

7. S. R. CARTER and S. RIMMERimmer, Adv. Funct. Mater. 14 (2004) 553.

8. Z. H. ZHANG, Y. J. LIU, Y. M. LONG, L. H. NIE, S. Z. YAO, Anal. Sci. 20 (2004) 291.

9. M. SUZUKI, M. YUMOTO, H. SHIRAI, and K. HANABUSA, (2005) Org. Biomolec. Chem. 3 (2005) 3073.

10. Y. HU, W. CHEN, J. CHEN, Z. ZHANG， (2003) Mater. Let. 57 (2003) 1312.

11. Y. HU, J. CHEN, W. CHEN and J. NING, (2003) Mater. Let. 58 (2003) 2911.

12. T. R. MATZELLE, D. A. IVANOV, D. LANDWEHR, L. A. HEINRICH, C. HERKT-BRUNS, R. REICHELT and N. KRUSE, (2002) J. Phys. Chem. B 106 (2002) 2861.

13. J. ZHANG and N. A. PEPPAS, J. Biomater. Sci. Poly. Ed. 13 (2002) 511.

14. S. HOU, Q. XU, W. TIAN, F. CUI, Q. CAI, J. MA and I-S. LEE, J. Neurosci. Meth. 148 (2005) 60.

15. J. L. NATION, Stain Technol. 58 (1983) 347.

16. W. CHEN, Y. YUAN and L. YAN (2000) Mater. Res. Bull. 35 (2000) 807. 
17. L. A. ESTROFF, L. LEISEROWITZ, A. ADDADI, S. WEINER and A. D. HAMILTON, (2003) Adv. Mater. 15 (2003) 38.

\section{Figure Legends}

1. TEM micrograph of NIP, the non-imprinted precursor. The scale bar in the lower left equals $50 \mathrm{~nm}$.

2. TEM micrograph of MIP 1. Arrows indicate cavities; scale bar in the lower left equals $50 \mathrm{~nm}$.

3. TEM micrograph of MIP 2. Arrows indicate cavities; scale bar in the lower left equals $50 \mathrm{~nm}$. 